\title{
Speckle Reduction in Imaging Projection Systems
}

\author{
Weston Thomas, Christopher Middlebrook \\ Electrical and Computer Engineering Department, Michigan Technological University, Houghton, USA \\ Email: whthomas@mtu.edu
}

Received October 10, 2012; revised November 15, 2012; accepted November 25, 2012

\begin{abstract}
Diffractive diffusers (phase gratings) are routinely used for homogenizing and beam shaping for laser beam applications. Another use for diffractive diffusers is in the reduction of speckle for pico-projection systems. While diffusers are unable to completely eliminate speckle they can be utilized to decrease the resultant contrast to provide a more visually acceptable image. Research has been conducted to quantify and measure the diffusers overall ability in speckle reduction. A theoretical Fourier optics model is used to provide the diffuser's stationary and in-motion performance in terms of the resultant contrast level. Contrast measurements of two diffractive diffusers are calculated theoretically and compared with experimental results. Having a working theoretical model to accurately predict the performance of the diffractive diffuser allows for the verification of new diffuser designs specifically for pico-projection system applications.
\end{abstract}

Keywords: Diffractive Diffusers; Speckle Contrast Reduction; Laser Pico-Projectors

\section{Introduction}

The observance of speckle in laser images is caused by the interference of the coherent source. Diffusers reduce speckle by decreasing the temporal and spatial coherence of the source. A diffuser has multiple cells each with different phase values. By rotating or vibrating the diffuser over a discrete time period the phase levels alter the coherence, thereby reducing the speckle contrast. The time-averaging of the speckle must occur over a discrete time smaller than the integration time of the human eye. Preceding investigations into the reduction of speckle contrast using rotational diffusers are well known and have had limited success [1-5]. The criterion for an effective diffuser is based on the calculation of the speckle contrast. Equation (1) gives the contrast, calculated by the standard deviation divided by the average intensity.

$$
C=\frac{\sqrt{\left\langle I^{2}\right\rangle-\langle I\rangle^{2}}}{\langle I\rangle}=\frac{\sqrt{\frac{\sum_{i=1}^{N}\left(I_{i}-\langle I\rangle\right)^{2}}{N-1}}}{\langle I\rangle}
$$

Figure 1 shows an example of a binary diffuser that is currently being used for reducing speckle in laser projections systems and will be discussed in detail later. This specific diffuser has circular symmetry for rotation purposes.

\section{Theory and Model}

This diffuser is modeled as a single scattering phase screen, written in the form of a transmission aperture, given by Equation (2) [6]. Figure 2(a) illustrates the placement of the diffuser in line with the initial beam and followed by the imaging screen in the $(x, y)$ plane which has the varying phase pattern across the diffuser surface as shown in Figure 3.

$$
t_{A}=\mathrm{e}^{\varphi(x, y)}
$$

The simulation will revolve around mimicking the operation of two distinct diffuser types: binary and grayscale. The binary diffuser is based on a hadamard matrix and thus contains only two distinct phase level sections. This generates sharp edges in the output image. The grayscale diffuser takes advantage of 64 discrete phase levels creating a smoother granular profile [7]. Both types are designed to reduce the visual degradation



Figure 1. Binary diffractive diffuser. 


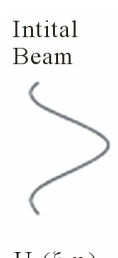

$\mathrm{U}_{\mathrm{G}}(\xi, \eta)$

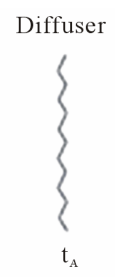

(a)
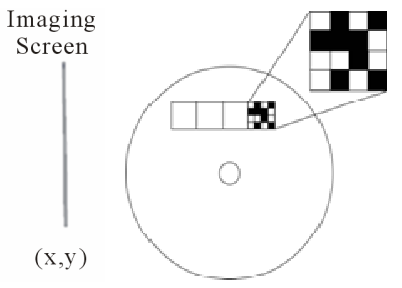

(b)

Figure 2. (a) Diffuser model layout; (b) Diffuser unit cell layout.

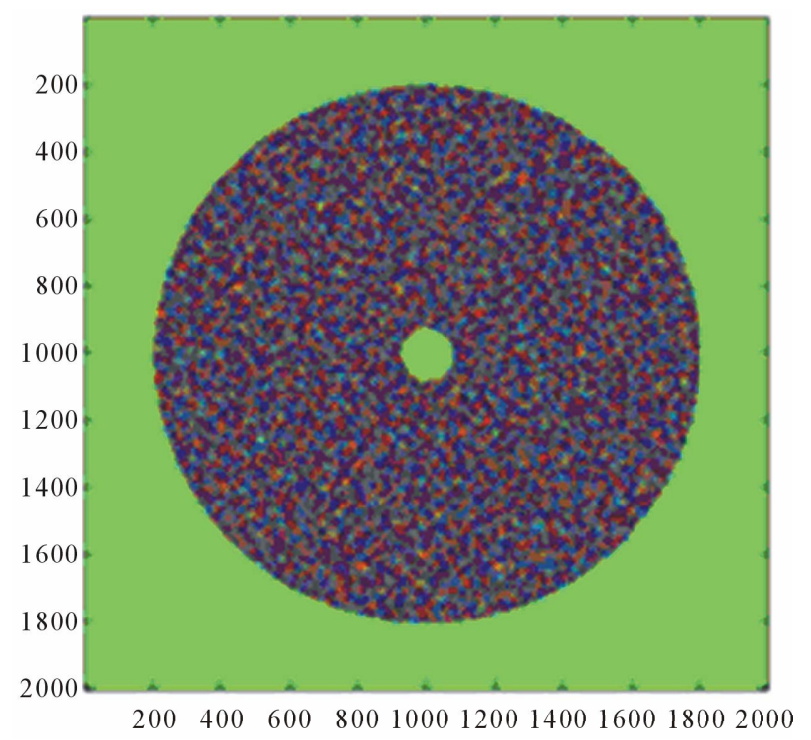

(a)

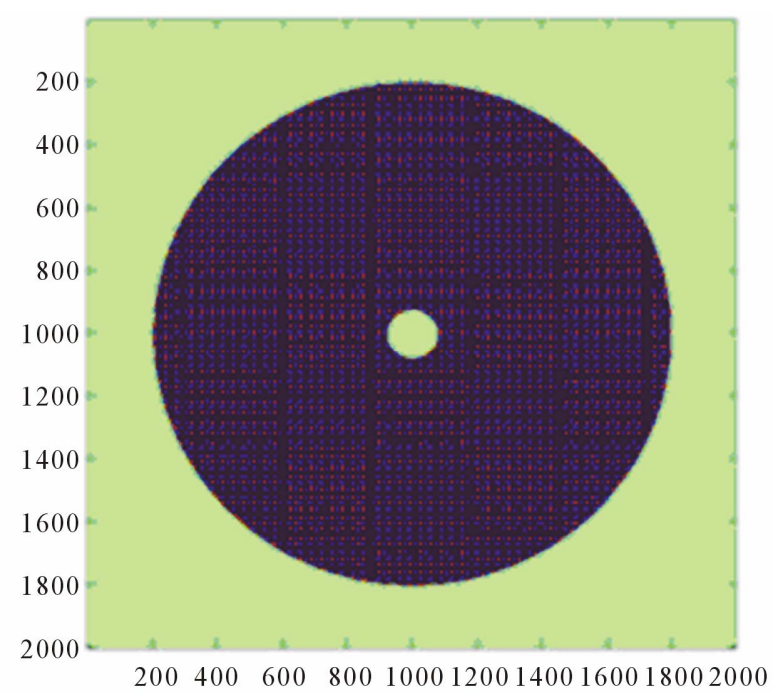

(b)

Figure 3. (a) Grayscale diffuser array; (b) Binary diffuser array.

caused by speckle through rotation. They are both modeled with an array of $2000 \times 2000$ pixels. The phase screen arrays are demonstrated in Figures 3(a) and (b).

Each diffuser is composed of individual cells replicated across the surface as demonstrated in Figure 2(b). The binary diffuser's unit cell is an order 16 Hadamard matrix while each Grayscale unit cell is a random organization of the 64 phase levels. These two unit cells are approximations of the physical diffusers that were used for experimental measurements but are not exact due to proprietary information.

Equation (3) demonstrates the linear size limitation given this array size and a $z$ depth of $2 \times 10^{4}$ pixels. Every individual phase element will consist of 25 pixels for each diffuser model. The minimum phase element size is 2.8 pixels for accurate sampling. This has been increased to be arranged evenly and symmetrically across the diffuser surface $[8,9]$. A distance mapping along the optical axis can be characterized along the z-axis. Every square pixel is related to a physical length by $2.5 \mu \mathrm{m}$. This mapping can be established for the propagation axis, z. This framing scale is maintained for all arrays unless distinctly noted on the axis of the image.

$$
\Delta x>\frac{\lambda z}{L}
$$

For the discrete version, $\Delta \mathrm{x}$ is the linear phase element size, $\lambda$ is the wavelength, $z$ is the propagation distance in pixels and $L$ represents the total physical side length of the array. The output from the aperture can be found by using a Fourier approximation for distance propagation such as the Fresnel equation [10]. Treating the mathematical computation as a linear system allows the separation of code to flow freely with the functional partitions. The system can be broken up into multiple parts as well based on the needs of the simulation. In this instance it is helpful to separate the Fourier optics propagation and the diffuser rotational program from the individual diffuser models allowing the diffuser models to be interchanged without rewriting large amounts of the simulation.

The initial beam is considered to be a collimated monochromatic Gaussian beam, as demonstrated in Figure 4, which is defined as [11]

$$
U_{G}(\xi, \eta)=\mathrm{e}^{-\frac{[(\xi+a) \times \Delta \xi]^{2}+[(\eta+b) \times \Delta \eta]^{2}}{w_{0}^{2}}}
$$

where the position $(a, b)$ is the center of the Gaussian beam, is the beam waist, and $(\Delta \xi, \Delta \eta)$ represents the physical size divided by the number of pixels in each direction. The laser wave front will be the size reference from which the rest of the simulation is measured. The xy-plane is the plane of incidence of the wave-front. The Gaussian beam is propagated through the diffuser in two steps. First the wave is multiplied by the transmission aperture, $t_{A}$ adding a phase displacement to the initial beam. The output of this calculation, shown in Equation (5), represents the field directly after the diffuser [12]. 


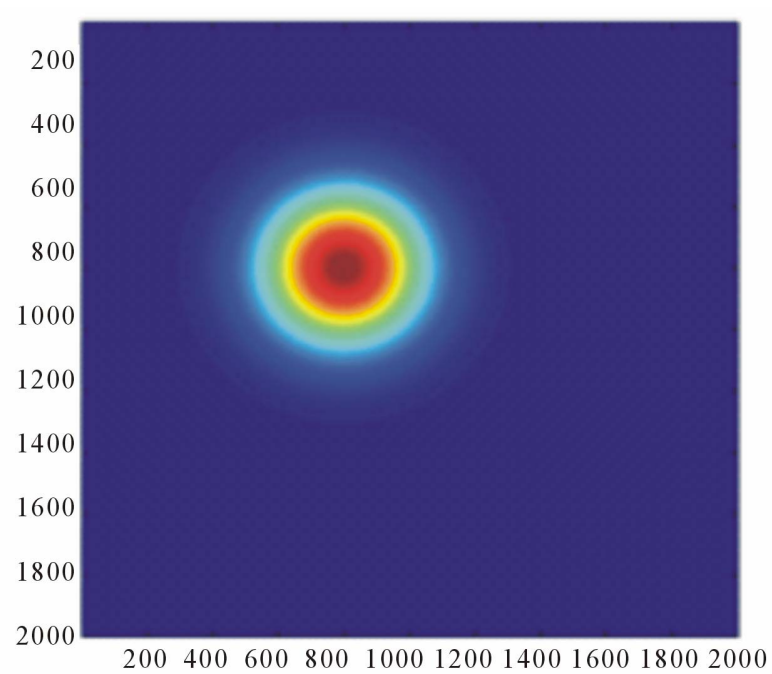

Figure 4. Gaussian beam profile.

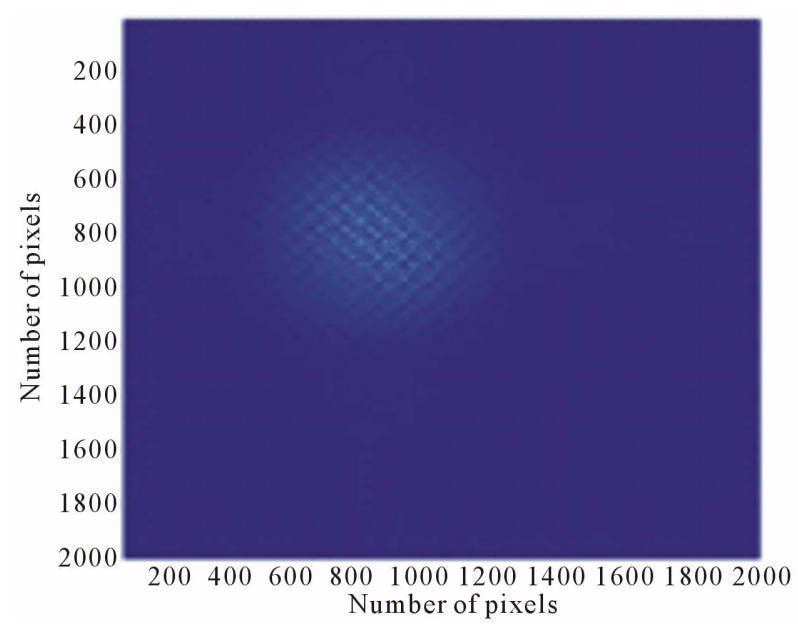

(a)

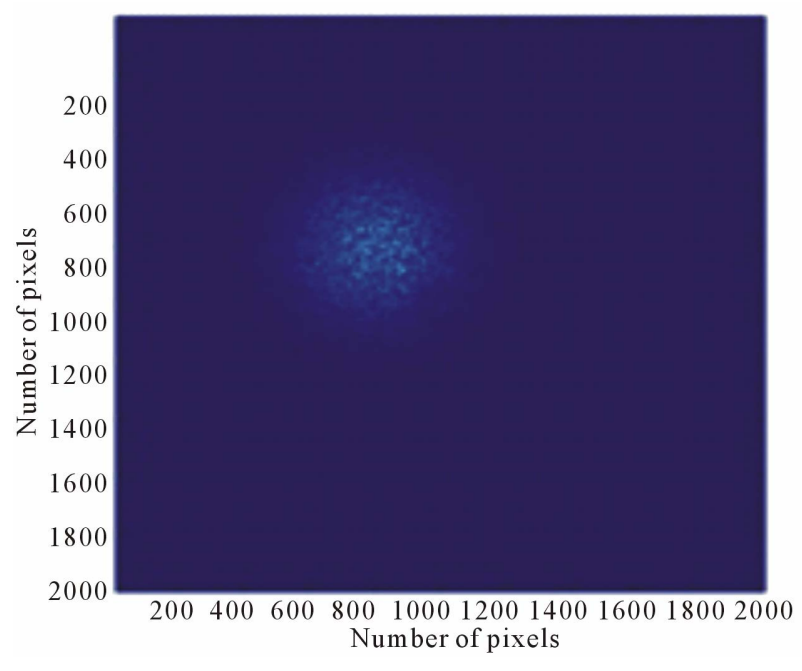

(b)

Figure 5. (a) Stationary binary diffuser; (b) Stationary grayscale diffuser.

$$
U_{1}(\xi, \eta)=U_{G}(\xi, \eta) \times t_{A}
$$

The second part involves the Fourier propagation of onto the $(x, y)$ image plane. This is accomplished through the Fresnel approximation of the Rayleigh-Sommerfield equation $[8,13]$. The Fresnel approximationis given in Equation (6) where $\lambda$ is the wavelength, $k$ is the wavenumber, $z$ is the propagation distance along the optical axis and $(\xi, \eta)$ is the aperture plane. The wavelength, once again, is chosen to be $535 \mathrm{~nm}$, representing a generic green laser diode as well as the center wavelength of the visible spectrum.

$$
U_{2}(x, y)=\frac{\mathrm{e}^{j k z}}{j \lambda z} \iint U_{1}(\xi, \eta) \mathrm{e}^{j \frac{k}{2 z}\left[(x-\xi)^{2}+(y-\eta)^{2}\right]} \mathrm{d} \xi \mathrm{d} \eta
$$

The final field image cannot be generated too close to the diffuser without causing quadratic errors in the exponential component. Using previous values for the diffuser aperture size and imaging array size, 1600 and 2000 pixels respectively, a proximity limit of 4500 pixels is calculated using Equation (7). This is considered the near-field region of measurement [13]. While the Fresnel integral does work in the far-field, the loss of image clarity due to beam expansion makes it less convenient to use and the model will refrain from approaching that region, defined by Equation (8) [13].

$$
\begin{aligned}
& z^{3} \gg \frac{\pi}{4 \lambda}\left[(x-\xi)^{2}+(y-\eta)^{2}\right]_{\max }^{2} \\
& z \gg \frac{k\left(\xi^{2}+\eta^{2}\right)_{\max }^{2}}{2}
\end{aligned}
$$

The final section of the simulation involves the rotation of the diffuser and the modeling of a camera or capture device. Using previous data, the diffuser can be sufficiently operated at $60(+/-0.5)$ revolutions per second [3]. In addition, the camera will operate at $30 \mathrm{~Hz}$, to mimic the eye's refresh rate or sampling time of around 23 frames per second [14]. To accomplish this the diffuser array will be rotated prior to propagation of the field $U_{1}(\xi, \eta)$. The formation of the final image is completed by addition of the individual fields while maintaining pixel position $(j, k)$ as characterized by Equation (9).

The final field is then normalized by the total number of images, $h$. The intensity image is then found by squaring the absolute value of the field, $U_{F}(x, y)$. A capture device integrates on the order of $10^{20}$ photons for a single image during a predetermined exposure time. In order to represent this, an approximation is resolved. This was completed through a series of Monte Carlo simulations. A generic noise pattern was created and then rotated a single revolution. The images were rotated and integrated at various degree increments ranging from 
0.01 degrees to 60 degrees. The resolution of the various images was compared against one another until the individual noise parameters were indistinguishable. This is the degree resolution from which the simulation will be completed. With the degree resolution defined at 0.75 the total number of images integrated to create a single frame is 480 . Comparing the speed of the diffuser at $60 \mathrm{~Hz}$ to the camera integration speed of $30 \mathrm{~Hz}$ it is identified that 2 rotations are completed for every single image frame. This will require at least 144,000 distinct images to represent a 10 second video capture.

\section{Results}

Calculations were made for the speckle contrast of stationary and moving diffuser images using Equation (1). The images for the stationary diffusers are shown below in Figures 5(a) and (b) for the binary and grayscale versions, respectively. The central spot of the diffuser is the zero-order diffractive mode. Most diffractive optical elements will consist of some form of zero-order mode and is considered to be the DC portion of the element. An optimum diffuser will minimize this zero-order and smooth out the overall output profile of the speckle. The first order of the element contains the majority of the incident power, $90 \%(+/-5 \%)$ as measured experimentally, as well as the highest speckle reductive properties. The speckle contrast is calculated for this first order spot of the diffuser image. The binary version has a contrast of 0.76 and the grayscale version has a contrast value of 0.74. These values correspond to data taken from intensity images of the simulation.

Close up versions of the central order of the diffuser image are shown in Figures 6(a) and (b). This is the physical representation of a focused image from the diffuser. They are $300 \times 300$ pixel array snapshots of the total diffuser image from above. This can be directly compared with bench top experiments taken using binary and grayscale diffusers with a $532 \mathrm{~nm}$ DPSS laser [3]. The camera used for the physical experiments had a 480 $\times 640$ pixel array. The stationary, focused diffuser images are shown in Figures 6(c) and (d). The contrast values for the bench top experiment are 0.77 for the binary diffuser and 0.68 for the grayscale version. The contrast values themselves are accurate to within $10 \%$. The images themselves, however, are not as comparable. The simulated versions have a more rigid structure while the bench top images are more fluid and organic, specifically related to the binary diffuser image. It is believed that this is a result of the inputs for the creation of the individual diffusers. The contrast results alone do provide enough accuracy for the simulation to be acceptable.

The simulation is finished by creating rotating variations of the diffuser patterns and propagating them onto

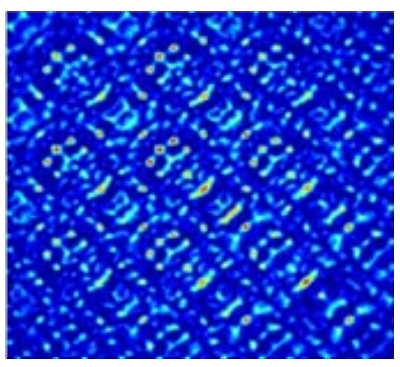

(a)

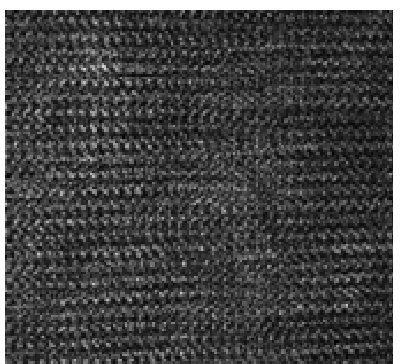

(c)

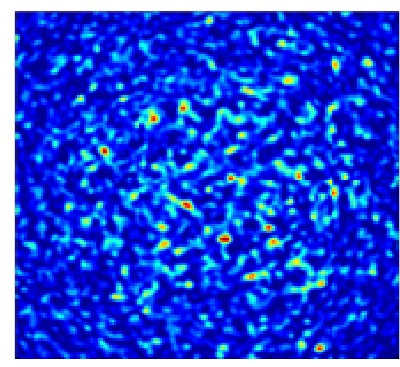

(b)

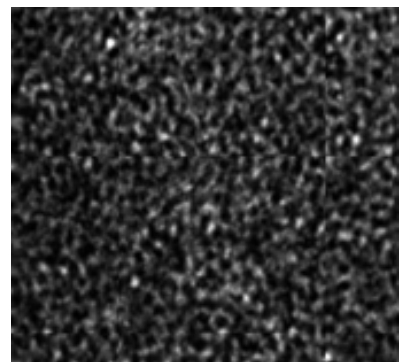

(d)
Figure 6. (a) Focused, stationary binary diffuser; (b) Focused, stationary grayscale diffuser; (c) Physical binary diffuser output intensity image; (d) Physical grayscale diffuser output intensity image.

the image plane. The parameters were set to mimic the rotational speed of the diffuser as $60 \mathrm{~Hz}$. The speckle was only measured for the central order as it was for the stationary diffuser images. The simulated images are shown in Figures 7(a) and (b). The calculated contrast values for the binary and grayscale images are 0.297 and 0.276 , respectively. The contrast values found in the bench top experiments were slightly less at 0.18 for the binary and 0.14 for the grayscale versions. This relates to a contrast difference of around $10 \%-13 \%$ contrast reduction between simulation and actual results.

The current version of this model focuses on a singlescattering approach [4]. While this approach provides a close approximation it does not fully agree with the physical diffuser scattering profile. This will be the main concentration for future work with the project. It is also assumed that a single polarization is incident on the diffusers and that the diffusers are polarization maintaining. Previous research showed that the polarization incident on the diffuser did not have an impact on the final contrast results [3]. This is an approximation that can be changed in the simulation to ensure the theoretical and experimental results match up. The simulation is still a good approximation as it does keep the contrast reduction rate between stationary and rotational the same at around $50 \%$ speckle decrease. This allows the simulation to become a useful tool for preliminary design work with theoretical diffuser shapes.

As mentioned earlier, the diffusers were illuminated 


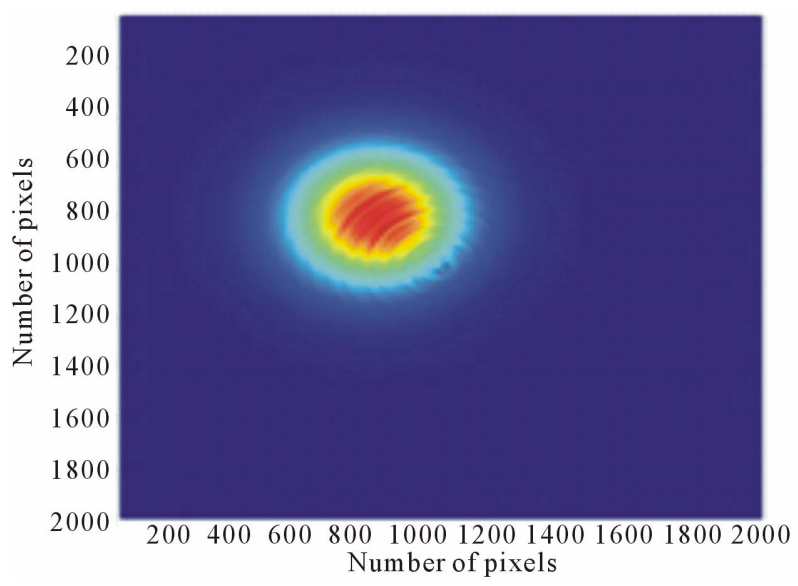

(a)

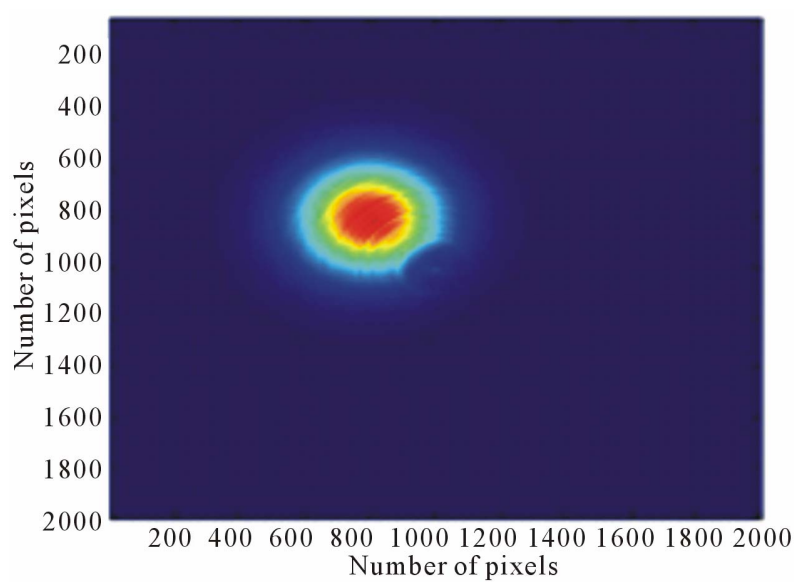

(b)

Figure 7. (a) Stationary binary diffuser; (b) Stationary grayscale diffuser.

with a coherent Gaussian source. All contrast values were measured without subtracting the Gaussian beam difference in the speckle. While uniform illumination speckle statistics are generally considered proper, it is by no means correct as standard laser diodes will have some sort of Gaussian shell and any speckle created from such a diode will have statistics correlating to the Gaussian laser beam. Verification was completed using the simulation model and comparisons were made between uniform illumination and a Gaussian source. Minor differences can be seen within Figure 6 but the primary result of the Gaussian illumination is the slight spreading of the speckle pattern and lower intensity within the center. Based on the speckle numbers this result is trivial and evens out across the final image plane, as demonstrated through Figures 8(a) and (b).

\section{RGB Integration}

In order to make full use of this model for a projector system more than one color must be combined for an

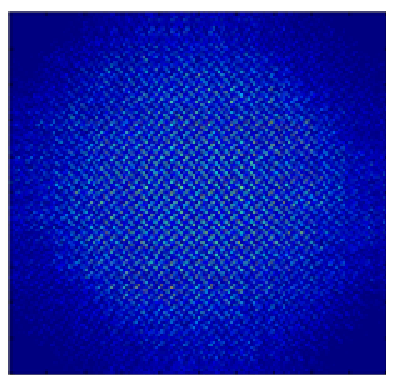

(a)

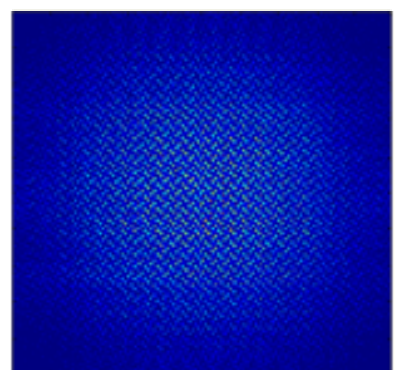

(b)
Figure 8. (a) Diffuser model with uniform illumination; (b) Diffuser model with Gaussian illumination.

image. Most diffusers are created for a single wavelength and errors result from using polychromatic light or multiple wavelengths. This problem will need to be solved before full implementation of diffusers into pico-projectors can be accomplished. In addition, integration of multiple colors into a single image can be difficult on its own due to the variation in the human eye's perception of colors [15]. Simulation will help to reduce problems in the creation and testing of multi-wavelength diffusers.

Beginning experiments were conducted to measure the wavelength dependence of the diffuser designs. The diffuser arrays were tested at wavelengths 635 and $450 \mathrm{~nm}$ to compare with the $535 \mathrm{~nm}$ beam results. The three wavelengths individual images for the grayscale diffuser simulation are shown in Figures 9(a)-(c). All three wavelength simulations were completed at the same distance from the diffuser along the optical axis. The obvious comparison between them is the expansion of the overall wave front. Speckle contrast measurements were taken for the central order of the diffuser. The contrast for the $450 \mathrm{~nm}$ image is 0.711 and is 0.65 for the $635 \mathrm{~nm}$ image.

The simulated images also allow the ability to combine the three wavelengths together into a single RGB image. Figure 9(d) illustrates this process and shows how the three distinct grayscale speckle patterns overlay onto one another. Current speckle calculations do not allow for 3 dimensional measurements and thus any calculation would simply be an average of the results for reach individual wavelength. In this case such an average gives a speckle contrast of 0.70 for the simulated grayscale diffuser. Also the colored images had to be normalized based on the visual response of the eye. Otherwise, the colors would appear out of sync and any speckle measurements would not align with visual representation of the speckle image [16]. A new measurement protocol will be needed to fully realize the speckle reduction ability of a diffuser design. Current investigations have led to comparisons between correlation times of diffusers and their resultant speckle contrast. 


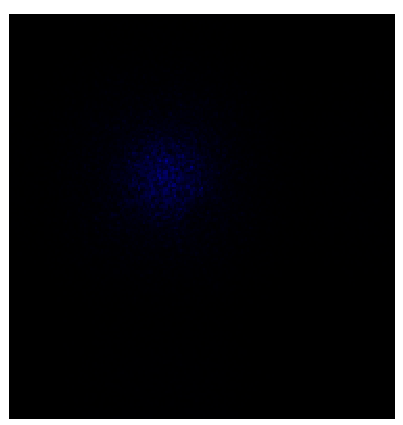

(a)

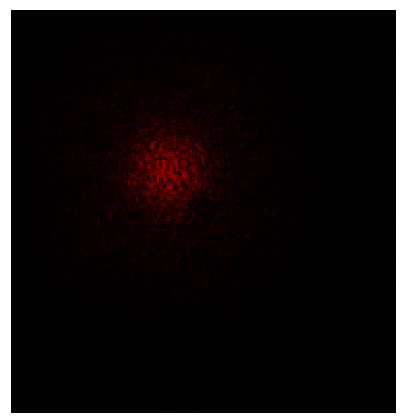

(c)

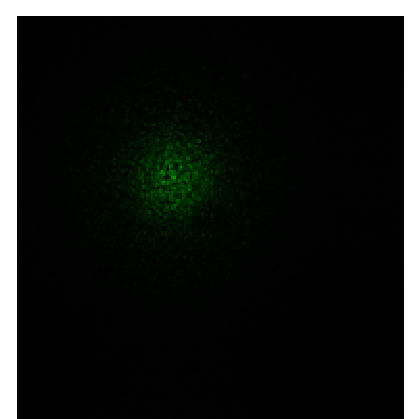

(b)

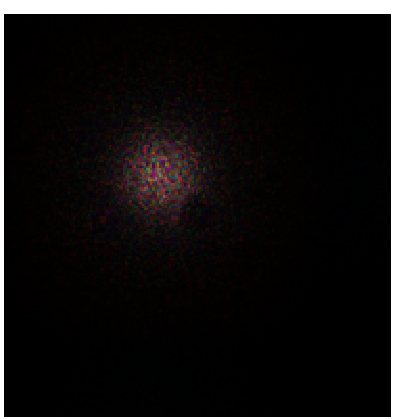

(d)
Figure 9. Simulated images of grayscale diffuser at (a) 450 nm; (b) $535 \mathrm{~nm}$; (c) $635 \mathrm{~nm}$; (d) Multi-wavelength combination.

\section{Conclusion}

A working mathematical model was developed to model diffractive diffusers for speckle reduction in imaging projection systems. The diffuser simulation was verified by comparing the results to experimental measured values for two distinct diffusers. The diffusers modeled have been shown to have contrast values ranging from $65 \%$ $77 \%$ and are accurate to within $10 \%$ of experimental results. Having the ability to perform multi-wavelength analysis of diffractive diffuser performance has also been shown with the model and verified with experimental results. The model will be used to aid in the design and performance of various diffractive diffuser designs for speckle reduction applications. This work provides the ability to quickly analyze multiple diffuser designs solely focused on creating a reduced speckle laser imaging projector system.

\section{REFERENCES}

[1] J. W. Goodman, "Speckle Phenomena in Optics: Theory and Applications," Roberts \& Company, Englewood, 2007, p. 387.
[2] J. I. Trisnadi, "Hadamard Speckle Contrast Reduction," Optics Letters, Vol. 29, No. 1, 2004, pp. 11-13. doi:10.1364/OL.29.000011

[3] W. Thomas, C. Middlebrook and J. Smith, "Laser Speckle Contrast Reduction Measurement Using Diffractive Diffusers," Proceedings of SPIE, Emerging Liquid Crystal Technological IV, Vol. 7232, 2009, 11 p.

[4] D. D. Duncan, S. J. Kirkpatrick and R. K. Wang, "Statistics of Local Speckle Contrast," Journal of the Optical Society of America, Vol. 25, No. 1, 2008, pp. 9-15. doi:10.1364/JOSAA.25.000009

[5] C. N. Kurtz, H. O. Hoadley and J. J. Depalma, "Design and Synthesis of Random Phase Diffuser," Journal of the Optical Society of America, Vol. 63, No. 9, 1973, pp. 1080 1092. doi:10.1364/JOSA.63.001080

[6] C. N. Kurtz, "Transmittance Characteristics of Surface Diffusers and the Design of Nearly Band-Limited Binary Diffusers," Journal of the Optical Society of America, Vol. 62 , No. 8, 1972, pp. 982-989. doi:10.1364/JOSA.62.000982

[7] J. W. Goodman, "Statistical Optics," Wiley Classics Library, John Wiley \& Sons, Hoboken, 2000, p. 550.

[8] Y. Nakayama and M. Kato, "Diffuser with Pseudorandom Phase Sequence," Journal of the Optical Society of America, Vol. 69, No. 10, 1979, pp. 1367-1372. doi:10.1364/JOSA.69.001367

[9] D. Voelz, "Computational Fourier Optics: A MATLAB Tutorial," SPIE Tutorial Texts, SPIE Press, Bellingham, 2011, p. 250.

[10] D. A. Gremaux, "Limits of Scalar Diffraction Theory for Conducting Gratings," Applied Optics, Vol. 32, No. 11, 1993, pp. 1948-1953. doi:10.1364/AO.32.001948

[11] J. E. Harvey, "Fourier Treatment of Near-Field Diffraction Theory," American Journal of Physics, Vol. 47, No. 11, 1979, pp. 974-980. doi:10.1119/1.11600

[12] J. T. Verdeyen, "Laser Electronics," In: J. N. Holonyak, Ed., Prentice Hall Series in Solid State Physical Electronics, 3rd Edition, Prentice Hall, Saddle River, 2000, p. 778.

[13] H. Loui, "Fourier Propagation, in Numerical Methods in Photonics Project 2004," University of Colorado, Boulder, pp. 1-5.

[14] J. W. Goodman, "Introduction to Fourier Optics," 3rd Edition, Roberts \& Company, Englewood, 2005.

[15] M. Livingstone, "Vision \& Art: The Biology of Seeing," Harry N. Abrams, New York, 2002.

[16] J. W. Tom, A. Ponticorvo and A. K. Dunn, "Efficient Processing of Laser Speckle Contrast Images," IEEE Transactions on Medical Imaging, Vol. 27, No. 12, 2008, pp. 1728-1738. doi:10.1109/TMI.2008.925081 